\title{
Ion energy distribution function in very high frequency capacitive discharges excited by saw-tooth waveform
}

Cite as: Phys. Plasmas 28, 103502 (2021); https://doi.org/10.1063/5.0061605

Submitted: 28 June 2021 • Accepted: 16 September 2021 • Published Online: 04 October 2021

(D) Sarveshwar Sharma, (Dishant Sirse, (D) Animesh Kuley, et al.
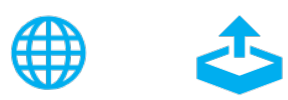

\section{ARTICLES YOU MAY BE INTERESTED IN}

A self-consistent method for solving ion diffusion and mobility coefficients

Physics of Plasmas 28, 103503 (2021); https://doi.org/10.1063/5.0060272

Numerical study on the production and transport of $\mathrm{O}$ and $\mathrm{OH}$ in a helium-humid air atmospheric pressure plasma jet interacting with a substrate

Physics of Plasmas 28, 103501 (2021); https://doi.org/10.1063/5.0058950

Negative ion of hydrogen in dense semi-classical plasmas: Stability and zero-energy resonances

Physics of Plasmas 28, 103504 (2021); https://doi.org/10.1063/5.0064894 


\title{
lon energy distribution function in very high frequency capacitive discharges excited by saw-tooth waveform
}

Cite as: Phys. Plasmas 28, 103502 (2021); doi: 10.1063/5.0061605

Submitted: 28 June 2021 • Accepted: 16 September 2021 .

Published Online: 4 October 2021

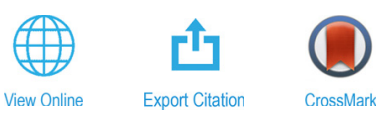

Sarveshwar Sharma, ${ }^{1,2}$ (D) Nishant Sirse, ${ }^{3, a)}$ (D) Animesh Kuley, ${ }^{4}$ (D) and Miles M Turner ${ }^{5}$ (D)

\begin{abstract}
AFFILIATIONS
${ }^{7}$ Basic Theory and Simulation Division, Institute for Plasma Research, Gandhinagar, Gujarat 382428, India

${ }^{2}$ Homi Bhabha National Institute, Anushaktinagar, Mumbai, Maharashtra 400094, India

${ }^{3}$ Institute of Science and Research, IPS Academy, Indore, Madhya Pradesh 452012, India

${ }^{4}$ Department of Physics, Indian Institute of Science, Bangalore, Karnataka 560012, India

${ }^{5}$ School of Physical Sciences and National Center for Plasma Science and Technology, Dublin City University, Dublin 9, Ireland
\end{abstract}

${ }^{\text {a) }}$ Author to whom correspondence should be addressed: nishantsirse@ipsacademy.org

\begin{abstract}
Tailoring the ion energy distribution function (IEDF) is vital for advanced plasma processing applications. Capacitively coupled plasma $(C C P)$ discharges excited using a non-sinusoidal waveform have shown its capability to control IEDF through the generation of plasma asymmetry and $D C$ self-bias. In this paper, we performed a particle-in-cell simulation study to investigate the IEDF in a symmetric capacitive discharge excited by a saw-tooth-like current waveform at a very high frequency. At a constant driving frequency of $27.12 \mathrm{MHz}$, the simulation results predict that the ion energy asymmetry in the discharge scales with the discharge current amplitude. A transition from a single narrow ion energy peak to a bi-modal type IEDF is observed with an increase in the current density amplitude. Further studies at a constant current density and varying the fundamental excitation frequency show that the ion energy asymmetry enhances with a reduction in the driving frequency. Increase in the plasma asymmetry and significant $D C$ self-bias at a lower driving frequency is observed to be one of the principal factors responsible for the observed asymmetry in the ion energy peaks. An investigation of $D C$ self-bias and plasma potential confirms that the powered electrode energy peak corresponds to the $D C$ self-bias with respect to the plasma potential, and the grounded electrode peak corresponds to the plasma potential. These results suggest that although lower driving frequency is beneficial for generating the discharge asymmetry and large $D C$ self-bias, a narrow low energy IEDF is plausible in very high frequency driven $C C P$ systems.
\end{abstract}

Published under an exclusive license by AIP Publishing. https://doi.org/10.1063/5.0061605

\section{INTRODUCTION}

Plasma technology plays a key role in microelectronic device fabrications, particularly in the processes including plasma enhanced chemical vapor deposition (PECVD) and plasma etching. ${ }^{1,2}$ In such processes, the ion energy distribution function (IEDF) on the wafer surface is one of the crucial parameters that drive the surface reactions. A radio frequency $(R F)$ capacitively coupled plasma $(C C P)$ system, which is a dominant plasma processing tool, mostly generates a broad bi-modal shape energy distribution in which the energy dispersion is defined by the ion transit time $\left(\tau_{i}\right)$ and frequency of the applied $R F$ field $(\omega){ }^{3,4}$ For the etching applications, as feature sizes are shrinking, one requires a narrow $I E D F$ for preventing the surface damage and to increase the selectivity across different materials. ${ }^{5}$ In the PECVD process, controlling the IEDF is vital for producing desired microstructures and film properties such as a translation from amorphous to microcrystalline structures.

In addition to controlling the shape of IEDF, an independent control of ion energy and ion flux at the substrate is highly desired. In a CCP discharge, one way to achieve this is to employ a second frequency either on the same electrode (dual-frequency CCPs) or on to the opposite electrode (two-frequency CCPs). ${ }^{7,8}$ In such configurations, the ion flux is governed by high frequency, while the low frequency controls the ion energy. Although multiple frequencies operating CCP discharges remain a better choice for an independent control of energy and flux, some of the earlier publications reported coupling between two frequencies, and therefore, undesired results 
are observed. ${ }^{9,10}$ Another method for achieving an independent control of ion flux and energy is the electrical asymmetry effect (EAE) as proposed by Heil et al. ${ }^{11,12}$ In this method, one can generate a $D C$ self-bias by changing the phase between a fundamental frequency and its first harmonic in a dual-frequency $C C P$ system. An experimental study by Schulze et al. ${ }^{13}$ verified that when using a variable phase angle between two frequencies, one could change the average ion energy approximately linearly by keeping the ion flux constant. Using this approach, the simulation results of Donkó et al. ${ }^{14}$ showed that it is possible to control the shape of IEDF at both powered electrode (PE) and grounded electrode (GE). In particular, higher moments of the IEDF could be varied with a change in the phase angle. ${ }^{15}$ However, further studies at different driving frequencies showed reduced ability of this method to control ion energy ranges at lower driving frequencies due to the secondary electron emissions. ${ }^{16}$ Choosing electrodes of dissimilar areas, geometrical asymmetry, and varied materials is the other way for the plasma asymmetry and $D C$ self-bias generation. ${ }^{17-20}$ A combination of the discharge voltage and driving frequency has been proposed as an alternative approach for an independent control of ion flux and energy. ${ }^{21}$

In recent years, the generation of electrically asymmetry using a non-sinusoidal waveform has emerged as a promising way to overcome the above challenges. ${ }^{22}$ Using such waveforms, one could generate an asymmetric plasma response, even in a geometrically symmetric CCP, by adjusting the no. of harmonics and phase between them. One of the examples is multi-harmonic waveform in which the plasma density/ion flux at one-electrode increases with the number of harmonics, while the average ion energy on the other electrode remains nearly constant. ${ }^{23-26}$ Another way to produce asymmetric plasma response is to use the temporally asymmetric waveforms such as saw-tooth-like waveforms. ${ }^{27-29}$ Such type of asymmetry is known as slope EAE. Using such waveforms, a vast disparity in the sheath expansion adjacent to the powered and grounded electrodes generates a strong asymmetry in the ionization rate, and thus, a flux asymmetry is developed. This effect was further analyzed and validated experimentally using phase resolved optical emission spectroscopy (PROES). ${ }^{30}$ It was demonstrated that the single frequency generated discharge shows the symmetric excitation rate at both electrodes, whereas the strong excitation rate asymmetry is observed when the number of harmonics is increased to generate a saw-tooth-like waveform. It was noticed that by increasing the number of harmonics in the waveform, i.e., turning into an ideal saw-tooth-like waveform further enhances the asymmetry in the discharge. ${ }^{27}$ Furthermore, reducing gas pressure has shown that the asymmetry disappears due to a transition from sheath edge ionization to bulk ionization. A study on the different gases revealed that saw-tooth-like waveform may cause strong asymmetry depending on the gas used. ${ }^{31}$

CCP discharges excited in a very high frequency (VHF) regime have shown several advantages over traditional CCP's excited at 13.56 MHz. In particular, an enhanced plasma density due to lower sheath impedance and reduced ion bombardment energy can be obtained. ${ }^{32,33}$ However, VHF plasma excitation generates plasma nonuniformities due to electromagnetic effects ${ }^{34,35}$ that limits their advantages for large area processing. Power deposition through multi, ${ }^{36-40}$ shaped $^{41-43}$ electrodes and waveform tailoring ${ }^{44}$ showed improvement in the plasma uniformities for VHF plasma excitation. In terms of discharge asymmetry produced by tailored waveform, changing the fundamental driving frequency has shown a drastic effect on it. In the case of saw-tooth-like voltage waveform, reducing the driving frequency from 54.24 to $1.695 \mathrm{MHz}$ has shown the shifting of ionization peak away from the powered electrodes. ${ }^{27}$ Lowering in driving frequency also reduces the ionization rate, and thus, the density decreases drastically. The combination of above two effects makes the discharge asymmetric as the driving frequency is reduced. Similar results were observed in the case of saw-tooth-like current waveform, ${ }^{29}$ where a strong asymmetry is observed at $13.56 \mathrm{MHz}$ when compared to $54.24 \mathrm{MHz}$ driving frequency. In addition to the discharge asymmetry, variation in the driving frequency has shown multiple ionization beams that are extended up to the opposite sheath and shown to modify the instantaneous sheath edge positions. ${ }^{29}$ The generation of multiple beams, which was also observed experimentally by Berger et al., ${ }^{45}$ is caused by the self-excitation of higher harmonics. The kinetic mechanism of such self-excitation of higher harmonics was described by Wiczek et al. ${ }^{46,47}$ Furthermore, the frequency of the sheath modulation was observed to increase with reducing driving frequency and, therefore, proposed as one of the parameters responsible for driving an enhanced plasma density in the discharge at lower fundamental driving frequency. ${ }^{29}$ On the other hand, higher beam energy at higher driving frequency was observed, which was due to the increased sheath velocity.

Above studies mostly focused on the electrical asymmetry and the generation of $D C$ self-bias in the discharge excited by nonsinusoidal waveforms. On the other hand, there exist very few studies of $I E D F$ in such discharge, particularly in VHF regime. One of the simulation studies performed by Schüngel et al. ${ }^{48}$ demonstrated that by exciting plasma, using a tailored waveform that consists of five harmonics could generate a control over the shape of IEDF. In particular, a single peak at low/medium energies within the IEDF could be generated and controlled by adjusting the parameters of the applied voltage waveform. We follow previous studies with the investigation of IEDF in the CCP discharge using an ideal saw-tooth-like current waveform at a very high frequency. In our previous work, ${ }^{29}$ we focused on the electric field non-linearity and higher harmonics generation in a symmetric CCP. In this paper, we extend our previous study ${ }^{29}$ and investigate the effect of current density amplitude on the IEDF at the powered and grounded electrodes in a symmetric CCP discharge. A saw-tooth-like current waveform at a fundamental driving frequency of $27.12 \mathrm{MHz}$ is considered for the present study. Additionally, we also investigate the effect of changing the fundamental driving frequency, from 13.56 to $54.24 \mathrm{MHz}$, on the shape of IEDF. Our simulation results predict that ion energy peaks correspond to the $D C$ self-bias and plasma potential. A narrow IEDF is observed at higher fundamental driving frequency.

This research article is organized as follows. The simulation technique, that is, based on the particle-in-cell/Monte Carlo collision (PIC/ MCC) methods and simulation parameters are described in Sec. II. The physical understanding and explanation of the simulation results are presented in Sec. III. In Sec. IV, the summary and conclusion are given.

\section{SIMULATION TECHNIQUE AND PARAMETERS}

The simulation technique is based on a particle-in-cell/Monte Carlo collision (PIC/MCC) methods. For the present study, we have 
used a well-tested and benchmarked $1 \mathrm{D} 3 \mathrm{~V}$, electrostatic, selfconsistent, PIC code. ${ }^{49,50}$ The code has been used extensively in previous studies, and details can be found in the literatures. ${ }^{51-63}$ As an input, a current waveform is chosen given by the following mathematical expression:

$$
J_{r f}(t)= \pm J_{0} \sum_{k=1}^{N} \frac{1}{k} \sin \left(k \omega_{R F} t\right) .
$$

In the above equation, the positive and negative signs resemble to "saw-tooth-down" and "saw-tooth-up" waveforms, respectively, $J_{o}$ is the amplitude of current density, and $\omega_{r f}$ is the fundamental angular $R F$.

An ideal saw-tooth waveform is desirable to simulate the asymmetry in the discharge, and therefore, the waveform should consist of higher number of harmonics. In our case, we have chosen $N=50$ harmonics, which are distributed in such a way to provide required peakto-peak current density amplitude for saw-tooth current waveform. Using such a large number of harmonics might cause difficulties in order to replicate the simulation results in the experiments due to the matching network issues. ${ }^{64}$ However, the current waveform consists of major contribution from the fundamental frequency, and other harmonics are below $10 \%$ (above $\mathrm{N}=4$ ) and even lower $<5 \%$ (above $\mathrm{N}=10$ ) for higher harmonics, and therefore, a comparison with experiments might not be an issue. We also checked the overall trend of simulation results for lower number of harmonics $(\mathrm{N}=4)$. It should be emphasized that the choice of current waveform is purely arbitrary as the purpose of this research work is to investigate the IEDF for sawtooth waveform and not to replicate or comparison with any specific experiments. One of the advantages for conducting simulation for current waveforms is the validation of the analytical models prediction, that is, available for the constant current conditions. ${ }^{65,66}$ In order to compare with the experiments, though exceptional, a current source can be obtained by implementing an $R F$ amplifier or generator with a very high output impedance in comparison to the plasma impedance. ${ }^{6,68}$ In this case, one can neglect the additional impedance of the plasma source, and consequently, current becomes the controlling parameter. The profile of the saw-tooth current waveform against sinusoidal waveform is shown in Fig. 1 for a fundamental driving frequency of $27.12 \mathrm{MHz}$.

The simulation is performed in argon gas at a fixed gas pressure of 5 mTorr, which is kept constant in the discharge region throughout the simulation. The discharge gap is $6 \mathrm{~cm}$. The saw-tooth-like current waveform is applied on the electrode at $0 \mathrm{~cm}$, and the other electrode at $6 \mathrm{~cm}$ is grounded. In the simulation, no external circuit with a capacitor is considered. Therefore, the $D C$ self-bias is generated due to the imbalance between electron and ion fluxes arriving at the electrodes caused by the applied waveform. The plasma chemistry considered in the simulation includes several particle-particle reactions such as ion-neutral (elastic, inelastic, and charge exchange) and electronneutral (elastic, inelastic, and ionization). The creation of two metastable states, $\mathrm{Ar} *$ and $\mathrm{Ar}^{*} *$, is also considered. These two lumped excited states of Ar, i.e., $\mathrm{Ar}^{*}$ (3p54s) $11.6 \mathrm{eV}$, and $\mathrm{Ar}^{*} *$ (3p54p), $13.1 \mathrm{eV}$, in uniform neutral argon gas background are considered with charged particles, viz., electrons and ions in the simulation. Important processes like multi-step ionization, metastable pooling, partial deexcitation, superelastic collisions, and further de-excitation with their

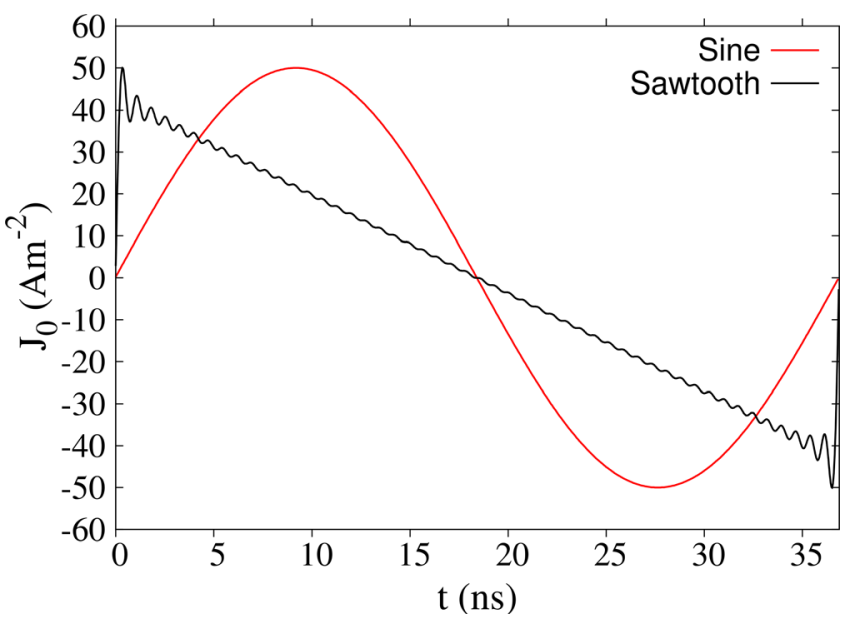

FIG. 1. Sinusoidal and saw-tooth current waveform at $27.12 \mathrm{MHz}$ fundamental driving frequency and current density amplitude of $50 \mathrm{~A} / \mathrm{m}^{2}$.

corresponding cross sections are taken from well-tested source and implemented into the simulation. ${ }^{53,58,69}$

The stability and accuracy criterion of PIC are achieved by selecting appropriate grid size $(\Delta x)$ and time step size $(\Delta t)$ that can resolve the Debye length $\left(\lambda_{d e}\right)$ and the electron plasma frequency, respectively $\left(f_{p e}\right){ }^{50}$ In the present study, the time step $(\Delta t)$ is of the order of $10^{-11} \mathrm{~s}$, and spatial grid size is of the order of $10^{-4} \mathrm{~m}$. It is assumed that both the electrodes have infinite dimension and are planar, equal in size, i.e., symmetric and parallel to each other. We have also considered that electrodes are perfectly absorbing. The secondary electron emission might play an important role even at a low gas pressure; ${ }^{70,71}$ however, in the present simulation, discharge voltage is low, ${ }^{29}$ and therefore, its effect can be ignored. The neutral gas is uniformly distributed with a fixed temperature of $300^{\circ} \mathrm{K}$ throughout the simulation. The temperature of ions is the same as the neutral gas temperature. The number of particles per cell taken here is 100 for all cases. All set of simulations run for more than $5000 R F$ cycles to achieve the steady state profile.

\section{RESULTS AND DISCUSSIONS}

We first investigate the plasma density in the discharge system by changing the current density amplitude at a fundamental driving frequency of $27.12 \mathrm{MHz}$. Figure 2(a) shows the time-averaged plasma (ion) density profile for different current density amplitudes ranging from 10 to $125 \mathrm{~A} / \mathrm{m}^{2}$. The corresponding time-averaged electron impact ionization rate, $e+A r \rightarrow 2 e+A r^{+}$, is plotted in Fig. 2(b). For the current set of operating parameters, it is clear from Fig. 2(a) that the plasma density at the center of the discharge is increasing from $\sim 7.8 \times 10^{14} \mathrm{~m}^{-3}$ at $10 \mathrm{~A} / \mathrm{m}^{2}$ to $\sim 2.3 \times 10^{16} \mathrm{~m}^{-3}$ at $125 \mathrm{~A} / \mathrm{m}^{2}$. On the other hand, the effective electron temperature $\left(T_{e}\right)$ at the center of the discharge (observed in simulation) decreases from $\sim 2.7 \mathrm{eV}$ at $10 \mathrm{~A} / \mathrm{m}^{2}$ to $\sim 2 \mathrm{eV}$ at $125 \mathrm{~A} / \mathrm{m}^{2}$, i.e., approximately $25 \%$ drop in $T_{e}$ is noticed. The corresponding ionization rate, displayed in Fig. 2(b), increases from $\sim 3.0 \times 10^{19} \mathrm{~m}^{-3} \mathrm{~s}^{-1}$ at $10 \mathrm{~A} / \mathrm{m}^{2}$ to $\sim 6.0 \times 10^{20} \mathrm{~m}^{-3} \mathrm{~s}^{-1}$ at $125 \mathrm{~A} / \mathrm{m}^{2}$. Furthermore, due to low gas pressure and longer mean free path, the ionization process mainly occurs in the plasma bulk. An increase in the ionization rate and, hence, the plasma density is mostly 

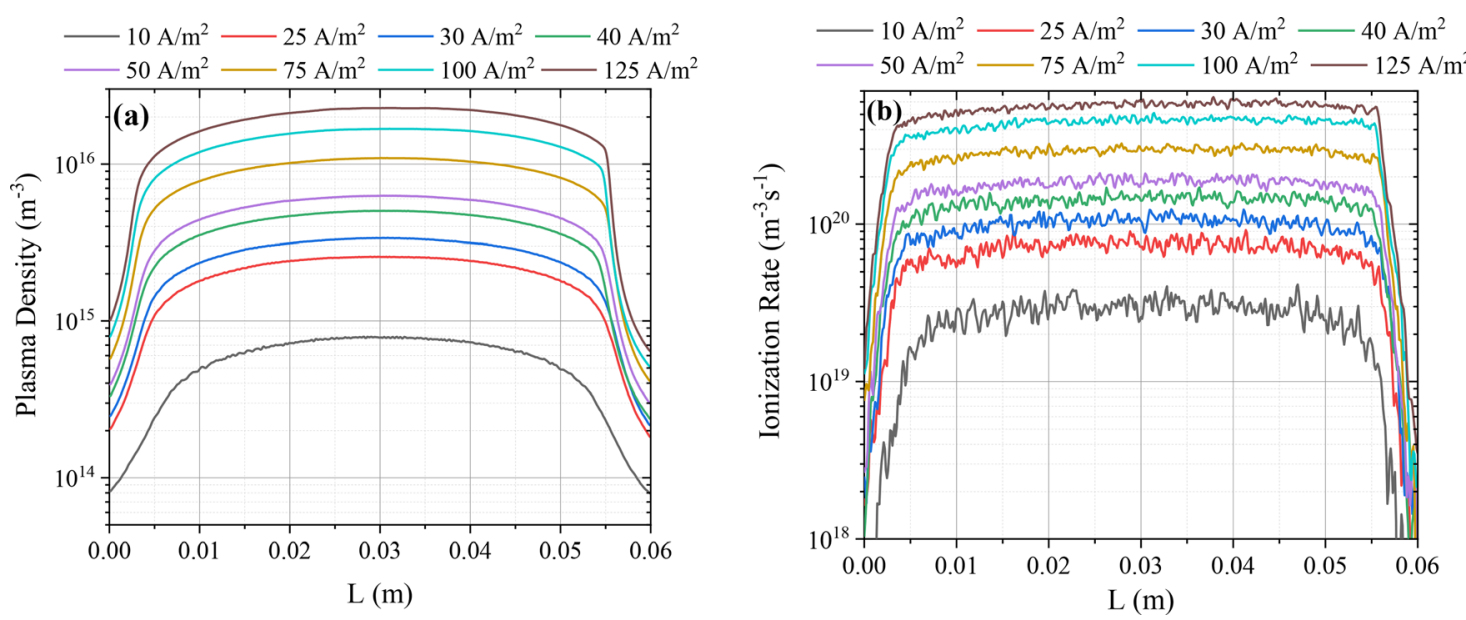

FIG. 2. The profile of (a) time-averaged plasma density and (b) the time-averaged ionization rate for different current densities from 10 to $125 \mathrm{~A} / \mathrm{m}^{2}$ at $27.12 \mathrm{MHz}$ driving frequency.

associated with the enhanced electron power deposition at higher current densities. At a low gas pressure, the electron heating mechanism in RF CCP discharge is overly complex because of nonlinear electron sheath interaction. Various processes such as stochastic and pressure/ambipolar heating have been proposed to elucidate the electron heating mechanism. ${ }^{1,72-76}$ It was further demonstrated that even the pressure/ambipolar heating mechanism is not always dominant at a low gas pressure due to the generation and collisionless transit of energetic electrons beam from near to the sheath edge that greatly attenuates the electron power absorption. ${ }^{77}$ The attenuation will be even prominent at a very high frequency because it produces multiple beams of energetic electrons. ${ }^{56-62}$ In the present case, the simulation predicts $\sim 20$ times increase in the electron heating, i.e., from $\sim 250 \mathrm{~W} / \mathrm{m}^{3}$ at $10 \mathrm{~A} / \mathrm{m}^{2}$ to $\sim 5000 \mathrm{~W} / \mathrm{m}^{3}$ at $125 \mathrm{~A} / \mathrm{m}^{2}$. A subsequent increase in the plasma density suggests that the energy gained by the electrons through interaction with the oscillating sheath dissipates mostly into the ionization processes (single/multi-step). As shown in Fig. 2(b), the ionization rate is increasing in proportion to the electron heating in the discharge, which confirm that the ionization is the dominant electron energy loss process.

Further analysis of the plasma density profile shows the generation of discharge asymmetry as the current density increases. Corresponding sheath width, estimated as where the electron sheath edge is at a maximum distance from the electrode and the quasineutrality condition breaks down, is nearly the same (approximately $5.5 \mathrm{~mm}$ ) at both electrodes for a current density of $10 \mathrm{~A} / \mathrm{m}^{2}$. However, as current density amplitude increases to $125 \mathrm{~A} / \mathrm{m}^{2}$, the plasma density profile becomes highly asymmetric. The sheath width at $125 \mathrm{~A} / \mathrm{m}^{2}$ is smaller $(\sim 2.7 \mathrm{~mm})$ near to the powered electrode and larger $(\sim 4.5 \mathrm{~mm})$ near to the grounded electrode. At higher current density, the asymmetry appears due to the fact that a large $D C$ self-bias is generated at the powered electrode. An emergence of $D C$ self-bias in a self-consistent manner from the simulation is attributed to the slope asymmetry of saw-tooth-like current waveform that generates ionization asymmetry in the discharge. ${ }^{29}$ Figure 3(a) displays the timeaverage potential profile for different current density amplitudes. Corresponding $D C$ self-bias at the powered electrode along with the plasma potential vs current density amplitude is shown in Fig. 3(b). As shown in Fig. 3(a), at $10 \mathrm{~A} / \mathrm{m}^{2}$, the time-averaged potential is nearly the same at both powered and grounded electrodes. The potential at the center of the discharge is $\sim 29 \mathrm{~V}$. As current density amplitude increases, positive $D C$ self-bias appears at the powered electrode. As shown in Fig. 3(b), both $D C$ self-bias and plasma potential scale with current density amplitude reaching to $\sim 130$ and $\sim 200 \mathrm{~V}$ at $125 \mathrm{~A} / \mathrm{m}^{2}$, respectively. The difference between the plasma potential and $D C$ selfbias is also increasing with the current density amplitude.

Figures 4(a) and 4(b) show the normalized IEDF at the powered electrode (PE) and grounded electrode (GE), respectively, for different current densities from 10 to $125 \mathrm{~A} / \mathrm{m}^{2}$. The fundamental driving frequency is $27.12 \mathrm{MHz}$. The energy of the different peaks observed in the $I E D F$ is also labeled. A clear asymmetry is observed in the ion energy at the powered and grounded electrodes. As shown in Figs. 4 (a) and 4 (b), at $10 \mathrm{~A} / \mathrm{m}^{2}$, the average ion energy is lowest ( $\sim 26 \mathrm{eV}$ at $\mathrm{PE}$ and $\sim 29 \mathrm{eV}$ at GE), and single energy peaks are observed with minimal asymmetry. As the amplitude of current density increases, the mean ion energy increases at both PE and GE. However, the ion energy at the GE is consistently higher when compared to the PE. The asymmetry between the energy peaks at the PE and GE continues to grow with an increase in current density amplitude. At higher current densities, such as 100 and $125 \mathrm{~A} / \mathrm{m}^{2}$, bi-modal energy peaks are observed with a large energy gap between PE and GE. An asymmetry in the energy peaks is attributed to $D C$ self-bias at the PE. A selfconsistent $D C$ self-bias from the simulation is due to an asymmetry in the spatiotemporal ionization rates and electron heating. ${ }^{29}$ As shown in Fig. 3(b), both plasma potential and DC self-bias increase with a rise in current density amplitude, and therefore, the asymmetry increases. For the present case, the average energy of the PE peaks is consistent with the difference between plasma potential and $D C$ self-bias $\left(V_{p}-V_{D C}\right)$. On the other hand, the average energy of GE energy peaks corresponds to the plasma potential $V_{p}$.

An increase in the average ion energy vs current density amplitude is attributed to a change in the sheath width and time-averaged sheath voltage. This is due to the fact that ion responds to timeaveraged potential. As shown earlier, the plasma is nearly symmetric, 

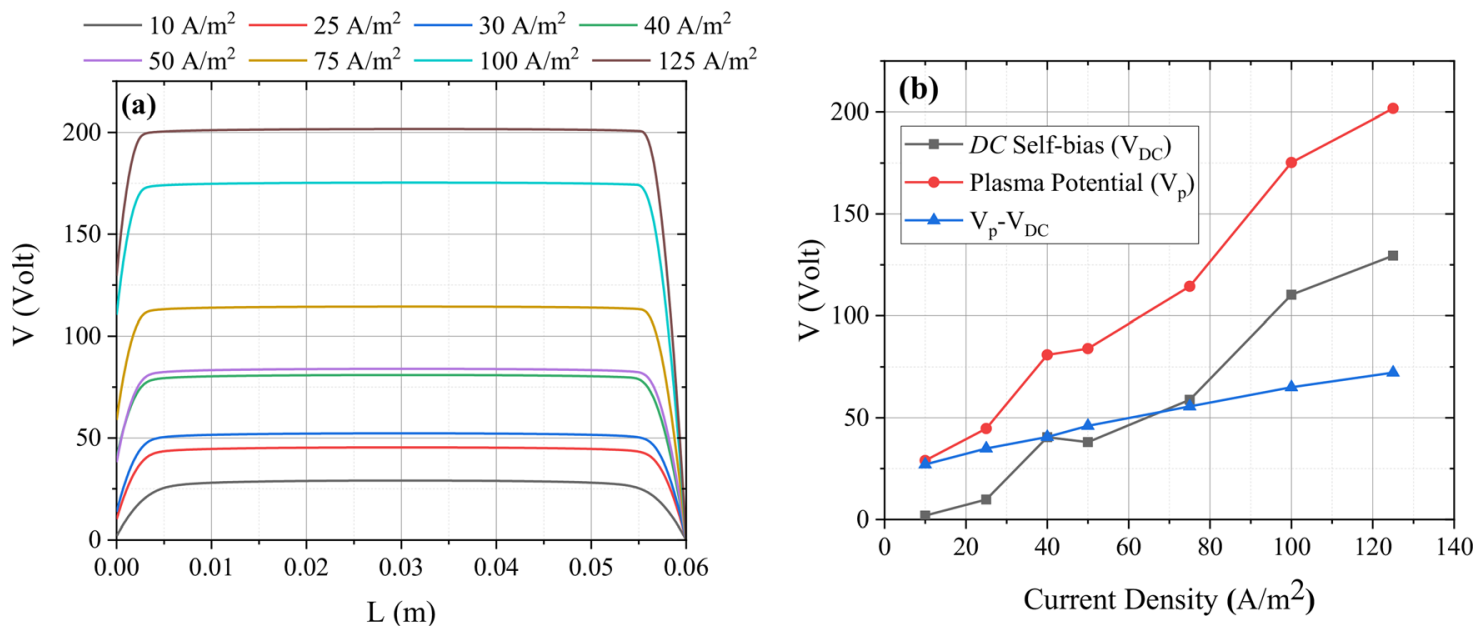

FIG. 3. (a) Time-averaged potential profile and (b) $D C$ self-bias at the powered electrode and potential at discharge center vs current density amplitude. The fundamental frequency of saw-tooth waveform is $27.12 \mathrm{MHz}$.

and sheath width is maximum $(\sim 5.5 \mathrm{~mm})$ at $10 \mathrm{~A} / \mathrm{m}^{2}$. A typical ion mean free path for the pressure regime is operating here, i.e., at $5 \mathrm{~m}$ Torr is nearly $0.6 \mathrm{~cm}$. A larger sheath width is responsible for the enhanced ion-neutral collisions. Furthermore, the time-averaged potential [Fig. 3(b)] at both PE and GE is lowest at $10 \mathrm{~A} / \mathrm{m}^{2}$, and therefore, the average ion energy arriving at the electrode decreases. As current density amplitude increases, time-averaged potential increases and sheath width decreases, which reduces the ion-neutral collisions within the sheath resulting to a higher average ion energy. On the other hand, the bi-modal behavior of IEDF at higher current densities corresponds to the ion transit time $\left(\tau_{i}\right)$ through $R F$ sheath. The ion transit time is defined as $\tau_{i}=3 s_{m} / v_{i} \propto V_{s}^{1 / 4} / n_{s}^{1 / 2}$, where $v_{i}, s_{m}, V_{s}$, and $n_{s}$ are the ion velocity in sheath, sheath width, averaged voltage drop across sheath, and density at the sheath edge, respectively. For a long ion transit time, $\tau_{i} \gg \tau_{r f}$, where $\tau_{r f}$ is the $R F$ period defined as $\tau_{r f}=2 \pi / \omega_{r f}$. However, for the short ion transit time, the condition is $\tau_{i} \ll \tau_{r f}$. ${ }^{1}$ So, the ion energy distribution is broad for short ion transit time case and highly peaked for the long ion transit time case. In the present case, the ion transit time at the powered electrode for a current density amplitude of $125 \mathrm{~A} / \mathrm{m}^{2}$ is of the order (30-50 ns) of $R F$ period $\left(\tau_{r f} \sim 37 \mathrm{~ns}\right)$, while at $10 \mathrm{~A} / \mathrm{m}^{2}$, it is $\sim 8$ times higher $(\sim 300 \mathrm{~ns})$ than $R F$ period. Therefore, one will expect a narrow IEDF at lower current density and bimodal at higher current density. For a constant driving frequency/RF period, the energy separation $\Delta E$ between two peaks of the bio-modal distribution is proportional to the sheath voltage and inversely proportional to the sheath width, i.e., $\Delta E \propto \sqrt{\left\langle V_{s h}\right\rangle} /\langle s\rangle,{ }^{3}$ where $\left\langle V_{s h}\right\rangle$ is the time-averaged sheath voltage and $\langle s\rangle$ is the timeaveraged sheath width. As observed in the present work, the sheath width decreases, and averaged sheath voltage increases with a rise in current density amplitude. Therefore, bi-modal peaks with large energy separation are observed at higher current density amplitudes.

The plasma asymmetry is greatly affected by the fundamental driving frequency due to a variation in the $R F$ period. Therefore, the role of the driving frequency is crucial in determining the shape of
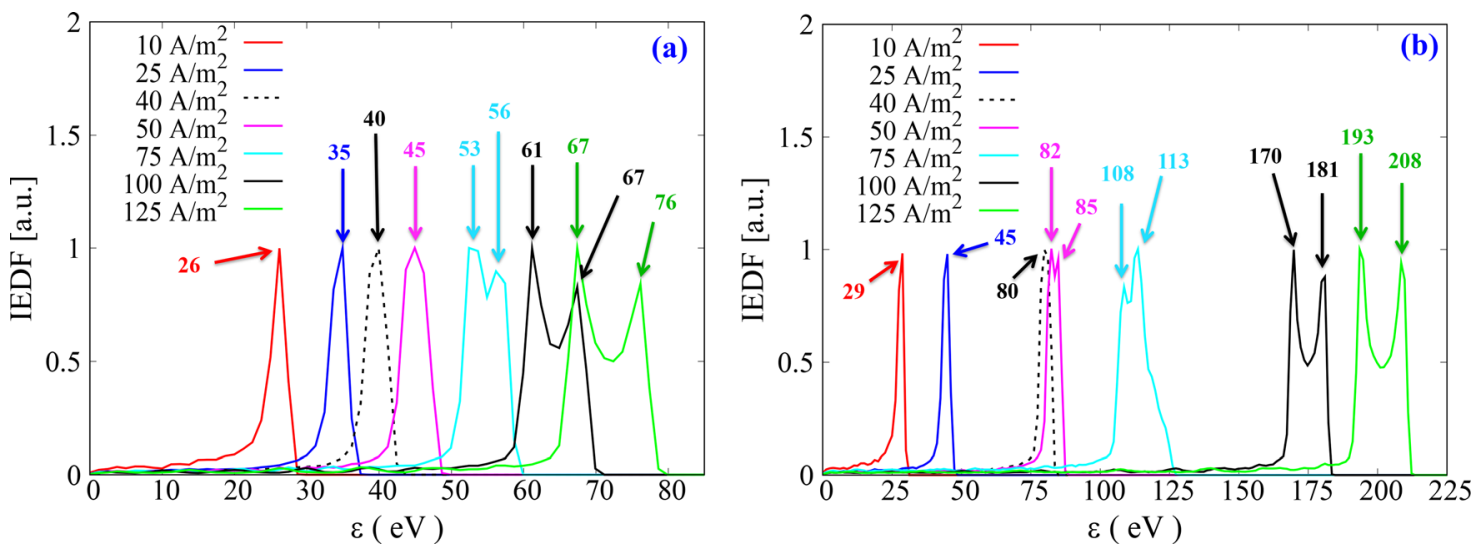

FIG. 4. IEDF at the (a) powered electrode and (b) grounded electrode for current density amplitudes from 10 to $125 \mathrm{~A} / \mathrm{m}^{2}$. The fundamental frequency of saw-tooth waveform is $27.12 \mathrm{MHz}$. 
IEDF. We investigate this by changing the fundamental driving frequency at a constant current density amplitude. Figure 5 shows the time-averaged plasma potential [Fig. 5(a)], IEDF at the powered electrode [Fig. 5(b)], and IEDF at the grounded electrode [Fig. 5(c)] for three different driving frequencies, $13.56,27.12$, and $54.24 \mathrm{MHz}$, at a fixed current density amplitude of $50 \mathrm{~A} / \mathrm{m}^{2}$. The simulations are also performed for lower number of harmonics $(\mathrm{N}=4)$ in order to verify the consistency of observed simulation trends. The results are plotted in Figs. 5(d)-5(f). As shown in Fig. 5(a), plasma is highly asymmetric at lowest driving frequency $(13.56 \mathrm{MHz})$, and a strong $D C$ self-bias $(\sim 210 \mathrm{~V})$ is observed at the powered electrode. As the driving frequency increases to $27.12 \mathrm{MHz}$, significant drop in the $D C$ self-bias is observed $(\sim 39 \mathrm{~V})$. At a $54.24 \mathrm{MHz}$ fundamental driving frequency, the plasma profile is nearly symmetric, and the $D C$ self-bias is nearly zero at the powered electrode. For $\mathrm{N}=4$, similar trend is observed, i.e., the $D C$ self-bias at the $\mathrm{PE}$ decreases with a rise in driving frequency
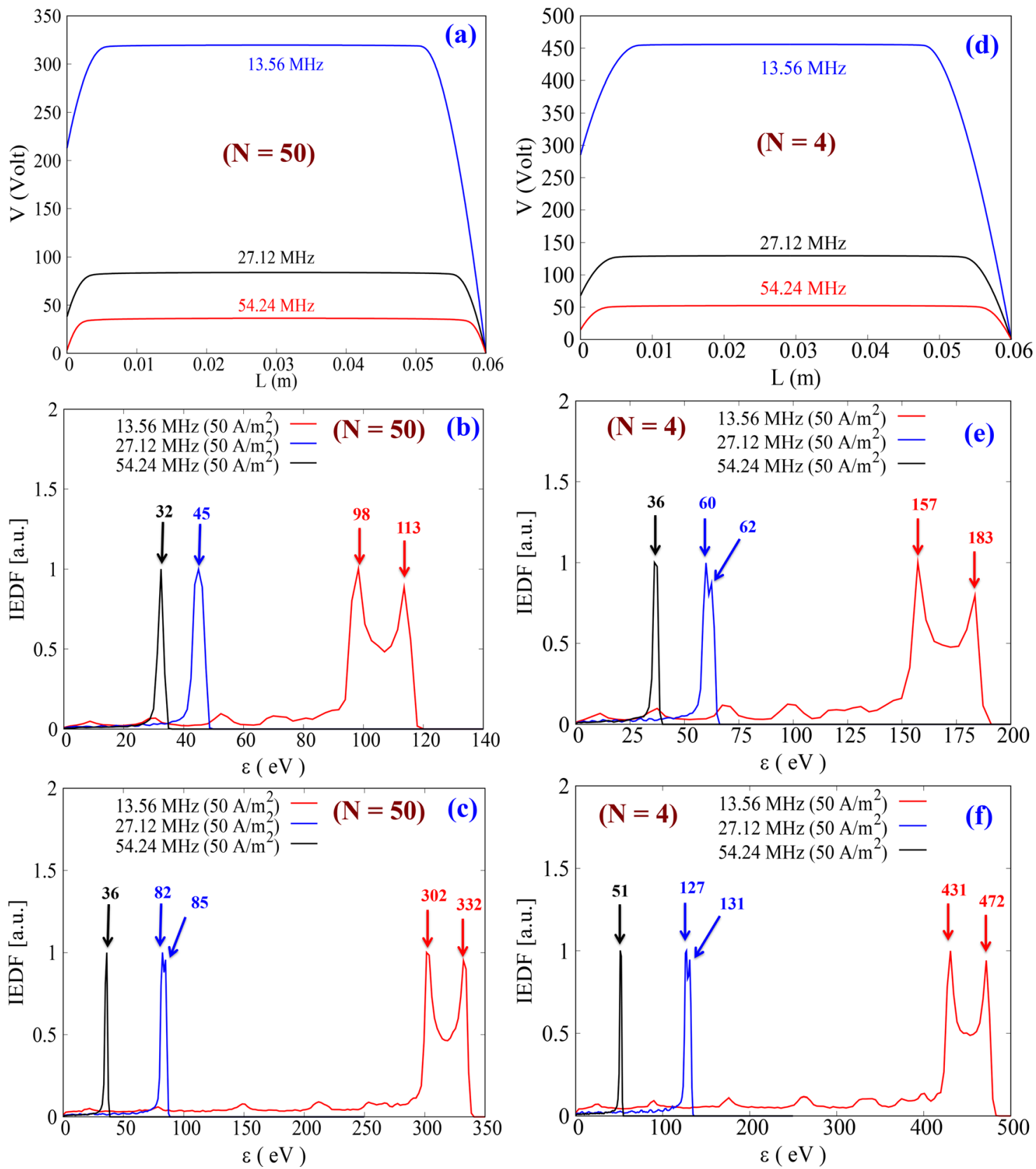

FIG. 5. Time-averaged plasma potential profile [(a) $N=50$ and (d) $N=4]$, IEDF at the powered electrode [(b) $N=50$ and (e) $N=4]$, and grounded electrode [(c) $N=50$ and (f) $\mathrm{N}=4$ ] for $13.56,27.12$, and $54.24 \mathrm{MHz}$ fundamental frequencies of a saw-tooth waveform at $50 \mathrm{~A} / \mathrm{m}^{2}$ current density amplitude. 
expect the absolute value is lower when compared to $\mathrm{N}=50$. The observed behavior is attributed to the spatiotemporal ionization asymmetry. As observed, ${ }^{29}$ at lower driving frequency $(13.56 \mathrm{MHz})$, the ionization near to the grounded electrode is higher when compared to powered electrode, whereas at higher driving frequency $(54.24 \mathrm{MHz})$, the ionization asymmetry reduces drastically.

Further analysis of IEDF shape presented in Figs. 5(b) and 5(c) depicts bi-modal distribution at both $\mathrm{PE}$ and GE for $13.56 \mathrm{MHz}$ driving frequency along with the strong ion energy asymmetry. The average energy of the PE peak is $\sim 106 \mathrm{eV}$ that corresponds to the $D C$ selfbias with respect to the plasma potential, whereas the average energy of the GE peak is $\sim 317 \mathrm{eV}$ that coincides with the time-averaged plasma potential. As the driving frequency increases to $27.12 \mathrm{MHz}$, bimodal peaks turned into single energy peaks at both PE and GE, and the ion energy asymmetry also decreases, i.e., PE peak is observed at $45 \mathrm{eV}$ and averaged ion energy at the GE is at $83.5 \mathrm{eV}$. Finally, at $54.24 \mathrm{MHz}$, narrow nearly symmetric energy peaks are observed at PE and GE with $\sim 36$ and $\sim 32 \mathrm{eV}$ ion energy, respectively. The conversion into single energy peaks as driving frequency increase is attributed to a drop in the time-averaged potential, as shown in Fig. 5(a). Furthermore, as driving frequency decreases, the $\mathrm{rf}$ period increase, and thus, broadening of the energy ion peak is anticipated, i.e., $\Delta E \propto\left\langle V_{\text {sh }}>/ \omega\right.$. At $13.56 \mathrm{MHz}$, strong asymmetry in the ion energy at the $\mathrm{PE}$ and GE is due to the generation of large $D C$ self-bias $(\sim 210 \mathrm{~V})$. Whereas, the $D C$ self-bias is lowest $(\sim 4 \mathrm{~V})$ at $54.24 \mathrm{MHz}$ fundamental driving frequency, which leads to a reduced asymmetry in the average ion energy. As displayed in Figs. 5(e) and 5(f), a transition in the shape of IEDF and ion energy asymmetry vs driving frequency is also true for smaller number of harmonics $(\mathrm{N}=4)$. These results support the validity of overall simulation outcomes, and thus, an experimental realization could be possible due to less complexity involved in generating saw-tooth-like waveform with lower number of harmonics.

\section{SUMMARY OF THE WORK AND CONCLUSIONS}

A study of plasma density, $D C$ self-bias/plasma potential, and $I E D F$ is performed using PIC simulation as a geometrically symmetric CCP discharge excited by a saw-tooth-like current waveform at a fixed gas pressure of $5 \mathrm{mTorr}$. For a fundamental driving frequency of 27.12 MHz, the plasma density increases with an increase in the current density amplitude. This is due to an overall increase in the electron heating and ionization rates. The time-averaged plasma potential observes to increase along with the formation of the $D C$ self-bias that scales linearly with the current density amplitude. At a lower current density amplitude, the plasma is nearly symmetric, and the IEDF at the powered and grounded electrodes shows a narrow low energy peak. As current density amplitude increases, bi-modal IEDF is observed with a large energy separation between the powered and grounded electrodes, i.e., the ion energy asymmetry increases. A close observation of IEDF suggests that the average ion energies at the PE and grounded electrodes correspond to the $D C$ self-bias and plasma potential. Changing the fundamental driving frequency of a saw-tooth current waveform leads to a drastic change in the DC self-bias, and hence, the ion energy asymmetry reduces at higher driving frequencies. A transition in the shape of IEDF from bimodal to a narrow single energy peak is observed with increasing driving frequency. For the simulation results, it is concluded that although lower fundamental driving frequency of a saw-tooth-like waveform is promising for generating discharge/ion energy asymmetry, a narrow low energy IEDF is mostly possible at a very high frequency due to a reduced rf period and decrease in the averaged sheath voltage.

\section{ACKNOWLEDGMENTS}

Dr. A. Kuley is supported by the Board of Research in Nuclear Sciences (BRNS Sanctioned No. 39/14/05/2018-BRNS), Science and Engineering Research Board EMEQ program (SERB Sanctioned No. EEQ/2017/000164), National Supercomputing Mission (NSM) (Ref. No.: DST/NSM/R\&D_HPC_Applications/2021/04), and Infosys Foundation Young Investigator grant.

The authors have no conflicts to disclose.

\section{DATA AVAILABILITY}

The data that support the findings of this study are available from the corresponding author upon reasonable request.

\section{REFERENCES}

${ }^{1}$ M. Lieberman and A. J. Lichtenberg, Principles of Plasma Discharges and Materials Processing (Wiley, New York, 2005).

${ }^{2} \mathrm{P}$. Chabert and N. Braithwaite, Physics of Radio Frequency Plasmas (Cambridge University Press, Cambridge, 2011).

${ }^{3}$ T. Panagopoulos and D. J. Economou, J. Appl. Phys. 85, 3435 (1999).

${ }^{4}$ E. Kawamura, V. Vahedi, M. A. Lieberman, and C. K. Birdsall, Plasma Sources Sci. Technol. 8, R45-R64 (1999).

${ }^{5}$ D. J. Economou, J. Vac. Sci. Technol. A 31, 050823 (2013).

${ }^{6}$ G. Bugnon, A. Feltrin, F. Meillaud, J. Bailat, and C. Ballif, J. Appl. Phys. 105, 064507 (2009).

${ }^{7}$ P. C. Boyle, A. R. Ellingboe, and M. M. Turner, J. Phys. D: Appl. Phys. 37, 697 (2004).

${ }^{8}$ T. Kitajima, Y. Takeo, Z. L. Petrovic, and T. Makabe, Appl. Phys. Lett. 77, 489 (2000).

${ }^{9}$ S. K. Karkari and A. R. Ellingboe, Appl. Phys. Lett. 88, 101501 (2006).

${ }^{10}$ T. Gans, J. Schulze, D. O'Connell, U. Czarnetzki, R. Faulkner, A. R. Ellingboe, and M. M. Turner, Appl. Phys. Lett. 89, 261502 (2006).

${ }^{11}$ B. G. Heil, J. Schulze, T. Mussenbrock, R. P. Brinkmann, and U. Czarnetzki, IEEE Trans. Plasma Sci. 36, 1404 (2008).

${ }^{12}$ B. G. Heil, U. Czarnetzki, R. P. Brinkmann, and T. Mussenbrock, J. Phys. D: Appl. Phys. 41, 165202 (2008).

${ }^{13}$ J. Schulze, E. Schüngel, and U. Czarnetzki, J. Phys. D: Appl. Phys. 42, 092005 (2009).

${ }^{14}$ Z. Donkó, J. Schulze, B. G. Heil, and U. Czarnetzki, J. Phys. D: Appl. Phys. 42, 025205 (2009).

${ }^{15}$ D. J. Coumou, D. H. Clark, T. Kummerer, M. Hopkins, D. Sullivan, and S. Shannon, IEEE Trans. Plasma Sci. 42, 1880 (2014).

${ }^{16}$ I. Korolov, Z. Donkó, U. Czarnetzki, and J. Schulze, J. Phys. D: Appl. Phys. 45, 465205 (2012).

17J. W. Coburn and E. Kay, J. Appl. Phys. 43, 4965 (1972).

${ }^{18}$ H. Köhler, J. W. Coburn, D. E. Horne, E. Kay, and H. Keller, J. Appl. Phys. 57, 59 (1985).

${ }^{19}$ M. A. Lieberman and S. E. Savas, J. Vac. Sci. Technol. A 8, 1632 (1990).

${ }^{20}$ T. Lafleur, P. Chabert, and J. P. Booth, J. Phys. D: Appl. Phys. 46, 135201 (2013).

${ }^{21}$ S. Sharma, A. Sen, N. Sirse, M. M. Turner, and A. R. Ellingboe, Phys. Plasmas 25, 080705 (2018).

${ }^{22}$ T. Lafleur, Plasma Sources Sci. Technol. 25, 013001 (2016).

${ }^{23}$ T. Lafleur and J. P. Booth, J. Phys. D: Appl. Phys. 45, 395203 (2012).

${ }^{24}$ P. A Delattre, T. Lafleur, E. V. Johnson, and J. P. Booth, J. Phys. D: Appl. Phys. 46, 235201 (2013).

${ }^{25}$ T. Lafleur, P. A. Delattre, E. V. Johnson, and J. P. Booth, Appl. Phys. Lett. 101, 124104 (2012). 
${ }^{26}$ T. Lafleur, P. A. Delattre, E. V. Johnson, and J. P. Booth, Plasma Phys. Controlled Fusion 55, 124002 (2013).

${ }^{27}$ B. Bruneau, T. Novikova, T. Lafleur, J. P. Booth, and E. V. Johnson, Plasma Sources Sci. Technol. 24, 015021 (2015).

${ }^{28}$ B. Bruneau, T. Novikova, T. Lafleur, J. P. Booth, and E. V. Johnson, Plasma Sources Sci. Technol. 23, 065010 (2014).

${ }^{29}$ S. Sharma, N. Sirse, and M. M. Turner, Plasma Sources Sci. Technol. 29, $114001(2020)$.

${ }^{30}$ B. Bruneau, T. Novikova, T. Lafleur, J. P. Booth, and E. V. Johnson, Phys. Rev. Lett. 114, 125002 (2015).

${ }^{31}$ B. Bruneau, T. Lafleur, T. Gans, D. O'Connell, A. Greb, I. Korolov, A. Derzsi, Z. Donkó, S. Brandt, E. Schüngel, J. Schulze, P. Diomede, D. J. Economou, S. Longo, E. Johnson, and J.-P. Booth, Plasma Sources Sci. Technol. 25, 01 LT02 (2016).

${ }^{32}$ A. A. Howling, J. L. Dorier, C. Hollenstein, U. Kroll, and F. Finger, J. Vac. Sci. Technol. A 10, 1080 (1992).

${ }^{33}$ M. Surendra and D. B. Graves, Appl. Phys. Lett. 59, 2091 (1991).

${ }^{34}$ M. A. Lieberman, J. P. Booth, P. Chabert, J. M. Rax, and M. M. Turner, Plasma Sources Sci. Technol. 11, 283 (2002).

${ }^{35}$ A. Perret, P. Chabert, J. Jolly, and J.-P. Booth, Appl. Phys. Lett. 86(1), 021501 (2005).

${ }^{36}$ C. Harvey, N. Sirse, C. Gaman, and A. R. Ellingboe, Phys. Plasmas 27, 110701 (2020).

${ }^{37}$ N. Sirse, C. Harvey, C. Gaman, and A. R. Ellingboe, J. Phys. D: Appl. Phys. 53, 335203 (2020).

${ }^{38}$ A. R. Ellingboe, "Plasma source," U.S. patent 7,342,361 B2 (2008).

${ }^{39}$ E. Monaghan, T. Michna, C. Gaman, D. O’Farrel, K. Ryan, D. Adley, T. S. Perova, B. Drews, M. Jaskot, and A. R. Ellingboe, Thin Solid Films 519(20), 6884 (2011)

${ }^{40}$ K. S. Kim, N. Sirse, K. H. Kim, A. R. Ellingboe, K. N. Kim, and G. Y. Yeom, J. Phys. D: Appl. Phys. 49(39), 395201 (2016).

${ }^{41}$ H. Schmidt, L. Sansonnens, A. A. Howling, C. Hollenstein, M. Elyaakoubi, and J. P. M. Schmitt, J. Phys. D: Appl. Phys. 95, 4559 (2004).

${ }^{42}$ L. Sansonnens and J. Schmitt, Appl. Phys. Lett. 82(2), 182 (2003).

${ }^{43}$ P. Chabert, J.-L. Raimbault, J.-M. Rax, and A. Perret, Phys. Plasmas 11, 4081 (2004).

${ }^{44}$ E. Schüngel, S. Mohr, J. Schulze, and U. Czarnetzki, Appl. Phys. Lett. 106, 054108 (2015).

${ }^{45}$ B. Berger, K. You, H.-C. Lee, T. Mussenbrock, P. Awakowicz, and J. Schulze, Plasma Sources Sci. Technol. 27, 12LT02 (2018).

${ }^{46}$ S. Wilczek, J. Trieschmann, D. Eremin, R. P. Brinkmann, J. Schulze, E. Schuengel, A. Derzsi, I. Korolov, P. Hartmann, Z. Donkó, and T. Mussenbrock, Phys. Plasmas 23, 063514 (2016).

${ }^{47}$ S. Wilczek, J. Schulze, R. P. Brinkmann, Z. Donkó, J. Trieschmann, and T. Mussenbrock, J. Appl. Phys. 127, 181101 (2020).

${ }^{48}$ E. Schüngel, Z. Donkó, P. Hartmann, A. Derzsi, I. Korolov, and J. Schulze, Plasma Sources Sci. Technol. 24, 045013 (2015).

${ }^{49}$ R. W. Hockney and J. W. Eastwood, Computer Simulation using Particles (Adam Hilger, Bristol, 1988).

${ }^{50}$ C. K. Birdsall, Plasma Physics via Computer Simulation (Adam Hilger, Bristol, 1991).
${ }^{51}$ M. M. Turner, A. W. Hutchinson, R. A. Doyle, and M. B. Hopkins, Phys. Rev. Lett. 76, 2069 (1996).

${ }^{52}$ P. C. Boyle, A. R. Ellingboe, and M. M. Turner, Plasma Sources Sci. Technol. 13, 493 (2004).

${ }^{53}$ L. Lauro-Taroni, M. M. Turner, and N. St. J. Braithwaite, J. Phys. D: Appl Phys. 37, 2216 (2004).

${ }^{54}$ M. M. Turner, Plasma Sources Sci. Technol. 22, 055001 (2013).

${ }^{55}$ J. Conway, S. Kechkar, N. O. Connor, C. Gaman, M. M. Turner, and S. Daniels, Plasma Sources Sci. Technol. 22, 045004 (2013).

${ }^{56}$ S. Sharma, N. Sirse, P. K. Kaw, M. M. Turner, and A. R. Ellingboe, Phys, Plasmas 23, 110701 (2016).

${ }^{57}$ S. Sarveshwar, N. Sirse, P. K. Kaw, M. M. Turner, and A. R. Ellingboe, in APS Gaseous Electronics Conference 2016 (APS, 2016).

${ }^{58}$ S. Sharma, N. Sirse, M. M. Turner, and A. R. Ellingboe, Phys. Plasmas 25 063501 (2018)

${ }^{59}$ S. Sharma, N. Sirse, A. Sen, J. S. Wu, and M. M. Turner, J. Phys. D: Appl. Phys. 52, 365201 (2019).

${ }^{60}$ S. Sharma, N. Sirse, A. Sen, M. M. Turner, and A. R. Ellingboe, Phys. Plasmas 26, 103508 (2019).

${ }^{61}$ S. Sharma, N. Sirse, A. Kuley, and M. M. Turner, Plasma Sources Sci. Technol. 29, 045003 (2020).

${ }^{62}$ S. Sharma, N. Sirse, A. Kuley, A. Sen, and M. M. Turner, J. Phys. D: Appl. Phys. 54, 055205 (2020).

${ }^{63}$ S. Sharma, S. K. Mishra, P. K. Kaw, and M. M. Turner, Phys. Plasmas 24, 013509 (2017).

${ }^{64}$ J. Wang, S. Dine, J.-P. Booth, and E. V. Johnson, J. Vac. Sci. Technol. A 37, 021303 (2019).

${ }^{65}$ M. M. Turner and P. Chabert, Appl. Phys. Lett. 104, 164102 (2014).

${ }^{66}$ M. M. Turner and P. Chabert, J. Phys. D: Appl. Phys. 50, 23LT02 (2017).

${ }^{67}$ V. A. Godyak, Soviet Radio Frequency Discharge Research (Delphic, Falls Church, VA, 1986).

${ }^{68}$ W. D. Qiu, K. J. Bowers, and C. K. Birdsall, Plasma Sources Sci. Technol. 12, 57 (2003).

${ }^{69}$ R. Shahid and M. J. Kushner, J. Appl. Phys. 82, 2805 (1997).

${ }^{70}$ B. Horvath, M. Daksha, I. Korolov, A. Derzsi, and J. Schulze, Plasma Sources Sci. Technol. 26, 124001 (2017).

${ }^{71}$ B. Horvath, J. Schulze, Z. Donko, and A. Derzsi, J. Phys. D: Appl. Phys. 51, 355204 (2018).

${ }^{72}$ M. M. Turner, Phys. Rev. Lett. 75, 1312 (1995).

${ }^{73}$ S. Sharma and M. M. Turner, Plasma Sources Sci. Technol. 22, 035014 (2013).

${ }^{74} \mathrm{~S}$. Sharma, "Investigation of ion and electron kinetic phenomena in capacitively coupled radio-frequency plasma sheaths: A simulation study," Ph.D. thesis (Dublin City University, Ireland, 2013).

${ }^{75}$ S. Sharma and M. M. Turner, J. Phys. D: Appl. Phys. 46, 285203 (2013).

${ }^{76}$ J. Schulze, Z. Donkó, T. Lafleur, S. Wilczek, and R. P. Brinkmann, Plasma Sources Sci. Technol. 27, 055010 (2018).

${ }^{77}$ M. Vass, S. Wilczek, T. Lafleur, R. P. Brinkmann, Z. Donkó, and J. Schulze, Plasma Sources Sci. Technol. 29, 085014 (2020). 